\title{
Absorbing Boundary Conditions for Free Surface Wave Simulations with a Panel Method
}

\author{
J. BROEZE AND J. E. RoMATE \\ Delft Hydraulics, P.O. Box 152,8300 AD Emmeloord, The Netherlands
}

Received November 22, 1989; revised January 29, 1991

\begin{abstract}
The numerical implementation and stability of first- and secondorder absorbing boundary conditions for simulating free surface gravity waves are considered. The free surface waves are solved with a panel method. The stability of the boundary conditions is proved, assuming certain properties of the integral operator. Arguments are given that support these assumptions. The theoretical results are confirmed in the test cases and the boundary conditions give low reflections for plane waves. (c) 1992 Academic Press, Inc.
\end{abstract}

\section{INTRODUCTION}

In this paper we will consider the numerical implementation and stability of absorbing boundary conditions for simulating surface gravity waves according to lincar theory with a potential flow model. These boundary conditions will be tested with a three-dimensional panel method.

In [9] absorbing boundary conditions are proposed that can be used on artificial boundaries in simulating gravity waves. These boundary conditions can be used to truncate large domains, while transmitting outgoing waves. These boundary conditions provide a well-posed problem. However, in numerical computations their discrete counterpart may lead to instabilities. Hence the stable implementation of these boundary conditions will be analyzed. Here these boundary conditions will be used in combination with a panel method. Furthermore, the quality of the boundary conditions for absorbing various waves will be tested.

The mathematical formulation of the problem is given in Section 2. In that section we will also discuss the panel method. The stability of the boundary conditions is analyzed in Section 3, and we will carry out the numerical tests in the fourth section.

\section{NUMERICAL ALGORITHM}

\subsection{Governing Equations}

In this section the solution procedure for the free surface problem is described.
Assuming that the fluid motion is described by a velocity potential $\phi(\nabla \phi=\mathbf{v})$, the free surface problem considered in this paper has the form [9]

$$
\begin{aligned}
\nabla^{2} \phi=0 & \text { in } \quad \Omega=\left\{\mathbf{x}=(x, y, z)^{\mathrm{T}} \mid x,\right. \\
y \in \mathbb{R},-h<z<0\} & \\
\frac{\partial \eta}{\partial t}=-\frac{\partial \phi}{\partial n} & \text { on } \quad z=0, \\
\frac{\partial \phi}{\partial t}=-g \cdot \eta & \text { on } \quad z=0, \\
\frac{\partial \phi}{\partial n}=0 & \text { on } \quad z=-h,
\end{aligned}
$$

where $\eta$ is the wave elevation and $\mathbf{n}$ denotes the inward normal. The depth $h$ is assumed to be constant.

Solid or artificial vertical boundaries truncate the fluid domain in the horizontal directions. On solid fixed boundaries the normal component of the velocity is zero. On artificial vertical boundaries, either a first-order absorbing boundary condition is imposed,

$$
\left(\cos (\alpha) \cdot \frac{\partial}{\partial t}-c \cdot \frac{\partial}{\partial n}\right) \phi=0 \quad(|\alpha|<\pi / 2)
$$

or a second-order absorbing boundary condition is used,

$$
a \cdot \frac{\partial^{2} \phi}{\partial t^{2}}-b \cdot \frac{\partial}{\partial t}\left(\frac{\partial \phi}{\partial n}\right)-c^{2} \cdot \frac{\partial^{2} \phi}{\partial s^{2}}+e \cdot \phi=0
$$

with

$$
\begin{aligned}
& a=\cos \left(\alpha_{1}\right) \cdot \cos \left(\alpha_{2}\right)+\frac{c}{c_{g}}, \\
& b=c \cdot\left(\cos \left(\alpha_{1}\right)+\cos \left(\alpha_{2}\right)\right), \\
& e=c^{2} \cdot\left(\frac{\omega^{2}}{c \cdot c_{g}}-k^{2}\right) .
\end{aligned}
$$


In these expressions $c$ is the phase velocity, and $c_{g}$ stands for the group velocity (see [9]). Equations (2.5) and (2.6) completely absorb waves with velocity $c$, at an angle of incidence $\theta= \pm \alpha$ (resp. $\theta= \pm \alpha_{1}$ or $\theta= \pm \alpha_{2}$ ) (see Fig. 1), while the reflection coefficient for waves from other directions is smaller than one (in general, Eq. (2.6) provides lower reflections than Eq. (2.5)).

The use of (2.6) in the end points leads to an unstable system. Hence we will use the first-order equation (2.5) in the corner points, or special second-order conditions for better reflection properties (see [9]):

$$
\begin{gathered}
\left(\sin \left(\alpha_{1}\right) \cdot \frac{\partial}{\partial t}-c \cdot \frac{\partial}{\partial s}\right)\left(\cos \left(\alpha_{2}\right) \cdot \frac{\partial}{\partial t}-c \cdot \frac{\partial}{\partial n}\right) \phi=0 \\
\left(s=s_{b}\right)
\end{gathered}
$$

and

$$
\begin{gathered}
\left(\sin \left(\alpha_{1}\right) \cdot \frac{\partial}{\partial t}+c \cdot \frac{\partial}{\partial s}\right)\left(\cos \left(\alpha_{2}\right) \cdot \frac{\partial}{\partial t}-c \cdot \frac{\partial}{\partial n}\right) \phi=0 \\
\left(s=s_{e}\right) .
\end{gathered}
$$

These boundary conditions absorb waves at an angle of incidence $\theta=\alpha_{1}$ or $\theta= \pm \alpha_{2}$. For waves from other directions from the interior of the domain, some reflection will occur, but with a reflection coefficient smaller than one. Both choices lead to a well-posed problem.

\subsection{Numerical Algorithm}

The numerical method used in our computations to solve the above equations is basically a panel method to solve Laplace's equation in combination with a Runge-Kutta method to integrate in time.

The panel method is based on Green's third identity, which is used to replace Laplace's equation by an integral equation over the boundary $S$ of the domain $\Omega$. Inserting $G(\mathbf{x}, \xi)=-1 /(4 \pi r)$ for the fundamental solution with $\mathbf{x}$ on the boundary $S$ provides an equation that involves only variables on $S$, making it possible to reduce the threedimensional problem of solving Laplace's equation on $\Omega$,

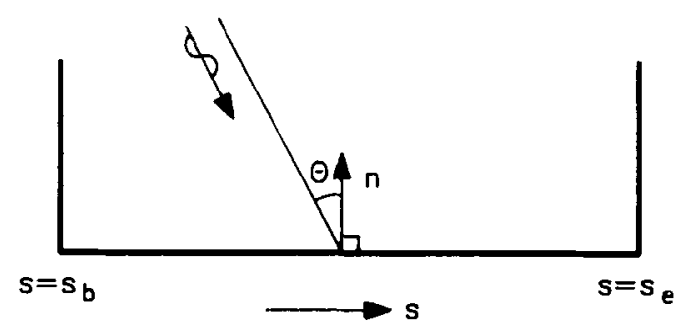

FIG. 1. Boundary with an approaching wave. to the two-dimensional problem of solving an integral equation on $S$ :

$$
\frac{1}{2} \cdot \phi(\mathbf{x})=\iint_{S}\left[\frac{\partial \phi}{\partial n_{\xi}}(\xi) \cdot G(\xi, \mathbf{x})-\phi(\xi) \cdot \frac{\partial G}{\partial n_{\xi}}(\xi, \mathbf{x})\right] d S_{\xi}
$$

( $\mathrm{x}$ on a smooth part of $S$ ).

We assume that the total boundary consists of a finite number of $C^{2}$-continuous sub-surfaces, each of which can be represented by $\mathbf{x}=\mathbf{x}(u, v),(u, v) \in[0,1] \times[0,1]$ in the computational domain. A sub-surface is divided into quadrilateral panels, with one collocation point per panel in its center in the computational domain. This point is used as an expansion point for the several functions defined on that panel.

Equation (2.9) is discretized by assuming quadratic variations of $\phi$ and linear variations of $\partial \phi / \partial n$ over each panel, and the panel surface was taken quadratic in $u$ and $v$ (linear source and quadratic dipole distribution; see [10] for details). Thus we have reduced the integral to an expression in terms of the values of $\phi$ and $\partial \phi / \partial n$ in the collocation points only. Implementing this with $\mathbf{x}$ in each collocation point results in $N$ equations with $2 N$ unknowns.

In each of the collocation points one of the unknowns can be eliminated by enforcing the boundary condition in that collocation point. The remaining $N$ unknowns can then be solved from the $N$ equations. Thus we need either $\phi$ or $\partial \phi / \partial n$ given in all $\mathbf{x}_{j}$. On the bottom, $\partial \phi / \partial n$ is prescribed, and this can be used directly.

On the free surface two first-order ordinary differential equations in time are given:

$\left(\begin{array}{l}\eta \\ \phi\end{array}\right)_{\imath}=\mathbf{u}_{t}=\left(\begin{array}{c}-\frac{\partial \phi}{\partial n} \\ -g \cdot \eta\end{array}\right)=\left(\begin{array}{rr}0 & 0 \\ -g & 0\end{array}\right) \mathbf{u}+\left(\begin{array}{c}-\frac{\partial \phi}{\partial n} \\ 0\end{array}\right)$

These conditions need no discretization in space and can be imposed directly in each collocation point in the free surface.

A discretization in time is needed. Starting from a known initial situation, the problem has to be solved in discrete time levels. Assuming that all variables are known in the collocation points at $t=t_{0}$, a Runge-Kutta scheme can be used to obtain $\phi\left(\mathbf{x}_{j}, t_{0}+\Delta t\right)$ and $\eta\left(\mathbf{x}_{j}, t_{0}+\Delta t\right)$. $\phi\left(\mathbf{x}_{j}, t_{0}+\Delta t\right)$ can serve as a boundary condition at the new time level $t_{0}+\Delta t$. The solution of $(\partial \phi / \partial n)\left(\mathbf{x}_{j}, t_{0}+\Delta t\right)$ from the integral equation then makes it possible to do a new time step.

The absorbing boundary conditions (2.8) and (2.10) must be discretized in time and in space. Not all discretizations 
lead to stable algorithms. Hence we will investigate the stability of the discrete model of this given (well-posed) initial boundary value problem (IBVP).

\section{STABILITY}

The basic tool for investigating the stability of a scheme is normal mode analysis. For pure initial value problems it is sufficient to consider modes which are purely oscillatory in space and to require that no such solutions may exist which grow in time. This is the Von Neumann condition for Cauchy stability.

For initial boundary value problems the question of stability is more difficult to answer. For finite difference models of hyperbolic problems a criterion based on normal mode analysis has been derived, which is similar to the uniform Kreiss condition (UKC) in well-posedness theory, providing a necessary and sufficient condition for the scheme to be stable in the sense of Gustafsson, Kreiss, and Sundstrom (GKS-stability), see [6].

Following [6], we consider the onc-dimensional firstorder equation

$$
u_{t}=a u_{x}+b, \quad a<0
$$

on $x, t \geqslant 0$, with initial and boundary conditions

$$
u(x, 0)=f(x), \quad u(0, t)=g(t) .
$$

A finite difference approximation $v_{j}^{n}$ of $u$ is computed in the grid points $\left(x_{j}, t_{n}\right), x_{j}=j \cdot \Delta x(j=0,1, \ldots)$ and $t_{n}=n \cdot \Delta t$ $(n=0,1, \ldots)$, with $\Delta x$ and $\Delta t$ constants. Let the finite difference scheme be a two-level scheme which can be written as

$$
\mathbf{v}^{n+1}=T\left(\mathbf{v}^{n}\right),
$$

thus expressing the values $\mathbf{v}^{n+1}$ in terms of previously computed variables. Boundary conditions are applied in $j=0$.

It is assumed that the solution $\mathbf{v}^{n}$ at any time level $n$ is in $l_{2}$, i.e.,

$$
\left\|\mathbf{v}^{n}\right\|_{x}^{2}=\Delta x \cdot \sum_{j=0}^{\infty}\left|v_{j}^{n}\right|^{2}<\infty,
$$

or that the solution is periodic.

If we also define the norms

$$
\left\|\mathbf{v}_{j}\right\|_{t}^{2}=\Delta t \cdot \sum_{n=0}^{\infty}\left|v_{j}^{n}\right|^{2}
$$

and

$$
\|\mathbf{v}\|_{x, t}^{2}=\Delta x \cdot \Delta t \cdot \sum_{j=0}^{\infty} \sum_{n=0}^{\infty}\left|v_{j}^{n}\right|^{2}
$$

the GKS-stability is basically defined as follows (for a precise definition, see [6, Definition 3.3]):

Definition 3.1 (GKS-stability). The finite difference scheme (3.3) is stable if constants $\alpha_{0} \geqslant 0$ and $K_{0}>0$ exist, such that for all $\alpha>\alpha_{0}$ the estimate

$$
\begin{aligned}
& \left(\frac{\alpha-\alpha_{0}}{\alpha \Delta t+1}\right) \cdot\left\|e^{-\alpha t} \cdot \mathbf{v}_{0}\right\|_{t}^{2}+\left(\frac{\alpha-\alpha_{0}}{\alpha \Delta t+1}\right)^{2} \cdot\left\|e^{-\alpha t} \cdot \mathbf{v}\right\|_{x, t}^{2} \\
& \quad \leqslant K_{0}^{2}\left[\left(\frac{\alpha-\alpha_{0}}{\alpha \Delta t+1}\right) \cdot\left\|e^{-\alpha(t+\Delta t)} \cdot g\right\|_{t}^{2}+\left\|e^{-\alpha(t+\Delta t)} \cdot b\right\|_{x, t}^{2}\right]
\end{aligned}
$$

holds.

This definition uses norms which are essentially integrals over $t$, which is very inconvenient, but for this stability definition, a necessary and sufficient condition exists. For this we introduce first the normal mode condition. Consider eigensolutions of the form

$$
v_{j}^{n}=e^{i k j \Delta x} \cdot e^{i \omega n \Delta t}=F^{j} \cdot G^{n},
$$

with $k, \omega \in \mathbb{C}$.

The Cauchy stability requires that the scheme admits no solutions (3.8) with $|F|=1$ and $|G|>1$ (Von Neumann condition). Furthermore, if the model would admit solutions (3.8) with $|F|<1$ and $|G|>1$, some initial error will grow unboundedly as well, spoiling the solution. Hence if these solutions are solutions of the interior scheme, the boundary condition should exclude them. This is the GodunovRyabenkii stability condition.

Solutions (3.8) with $|F|<1$ and $|G|=1$ are called evanescent modes. The occurrence of these solutions is less of a problem than the above case with $|G|>1$, since an instability originating at the boundary will decay exponentially in the $x$-direction. Yet it is desirable to exclude these solutions as well.

Gustafsson, Kreiss, and Sundstrom show that for stability it is, furthermore, necessary to exclude modes (3.8) with $|F|=|G|=1$ as a solution of the model, which are limiting cases of solutions with $|F|<1$ and $|G|>1$. Basically this last condition states that waves that radiate from the boundary into the interior due to a group velocity pointing into the interior, should not be admitted.

We will summarize these requirements in the following condition, which we will name the normal mode condition:

Definition 3.2 (Normal mode condition, NMC). A scheme satisfies the normal mode condition if no solutions of the form (3.8) are admitted with,

$$
|F|=1, \quad|G|>1 \quad \text { (Von Neumann) }
$$


or

$$
|F|<1, \quad|G|>1 \quad \text { (Godunov-Ryabenkii) }
$$

or

$|F|<1, \quad|G|=1 \quad$ (evanescent modes)

or

$|F|=1, \quad|G|=1, \quad$ which perturb to $|F|<1$ for $|G|>1$.

Now the following theorem holds:

THEOREM 3.1. A finite difference model of (3.1)-(3.2) is $G K S$-stable if and only if the scheme satisfies the NMC.

Note that this condition may not be sufficient for other stability definitions.

Now, consider again the free surface problem. A rigorous stability theory for discretizations of this kind of IBVPs has not been developed yet. But since (3.9) and (3.10) are necessary conditions for any practical stability concept and (3.11) and (3.12) are also required in many cases, the NMC is a useful condition for stability in any case. Therefore, we will check whether our discrete model satisfies the NMC, adapted to this problem.

Because the classical fourth-order Runge-Kutta scheme, which we use for the time integration, has a stability region which contains the intersection of a (rather large) neighborhood of zero and the negative half-plane, it is sufficient to consider only the semi-discrete case, in which only space-like operators are replaced by their discrete counterparts. When stability is obtained in the semi-discrete case, the fully discrete model is also stable if the time-step used is small enough. Thus we consider eigensolutions of the form

$$
\begin{aligned}
\phi_{m n l} & =\exp \left(i\left(k_{x} m \Delta x+k_{y} n \Delta y+k_{z} l \Delta z\right)\right) \cdot e^{\beta t} \\
& =F_{x}^{m} \cdot F_{y}^{n} \cdot F_{z}^{l} \cdot G^{t},
\end{aligned}
$$

and investigate whether the discrete model admits solutions that violate the NMC.

Our stability analysis will proceed along the following path. First wc will investigate the Cauchy stability of the discretization of the initial value problem (2.10). Then we include a boundary at $x=0$ in our considerations and investigate the stability of the discrete model (with absorbing boundary conditions) for the half-space problem by means of the NMC. We will finish the analysis by considering the corner problem.

\subsection{Stability of the Unbounded Free Surface}

Consider the initial value problem (2.10). Assuming that the equations are solved exactly in time, a numerical solu- tion $\left(\eta_{m n}, \phi_{m n}\right)=\mathbf{u}_{m n}=\mathbf{u}\left(x_{m}, y_{n}, 0\right)$ has to be determined at the discrete points $\left(x_{m}, y_{n}, 0\right)=(m h, n h, 0)$ at the surface. Writing the discrete counterpart of (2.10) as

$$
\frac{\partial}{\partial t} \mathbf{u}_{m n}=\left(\begin{array}{cc}
0 & -A \\
-g I & 0
\end{array}\right) \mathbf{u}_{m n}=B \mathbf{u}_{m n}
$$

with $A$ the discrete approximation of $\partial / \partial n=-\partial / \partial z$, this equation can be Fourier transformed using the discrete Fourier transform

$$
\begin{aligned}
\hat{f}\left(k_{x}, k_{y}\right)= & F_{d}\left(f_{m n}\right) \\
= & h^{2} \sum_{m=-\infty}^{\infty} \sum_{n=-\infty}^{\infty} f_{m n} \\
& \times \exp \left(-i k_{x} x_{m}-i k_{y} y_{n}\right) .
\end{aligned}
$$

The inverse transform is given by

$$
\begin{aligned}
f_{m n}= & F_{d}^{-1}\left(\hat{f}\left(k_{x}, k_{y}\right)\right) \\
= & \frac{1}{2 \pi} \int_{-\pi / h}^{\pi / h} \int_{-\pi / h}^{\pi / h} \hat{f}\left(k_{x}, k_{y}\right) \\
& \times \exp \left(i k_{x} x_{m}+i k_{y} y_{n}\right) d k_{x} d k_{y} .
\end{aligned}
$$

System (3.14) gives the transformed system

$$
\frac{\partial}{\partial t} \hat{\mathbf{u}}_{m n}=\left(\begin{array}{rr}
0 & -\hat{A} \\
-g & 0
\end{array}\right) \hat{\mathbf{u}}_{m n}=\hat{B} \hat{\mathbf{u}}_{m n} .
$$

For the eigenvalues $\beta$ of $\hat{B}$ we have

$$
\begin{gathered}
\beta_{j}=\sqrt{g|\hat{A}(\mathbf{k})|} \cdot \exp (i(\angle \hat{A}(\mathbf{k})+2 j \pi) / 2), \\
j=0,1,
\end{gathered}
$$

in which $\angle A$ denotes the argument of $\hat{A}(\mathbf{k})$, and hence the eigensolutions are

$$
\begin{aligned}
\mathbf{u}_{m n}(t)_{j}= & \mathbf{v}_{j} \cdot \exp \left(i\left(k_{x} x_{m}+k_{y} y_{n}\right)\right) \\
& \times \exp \left(\operatorname{Re}\left(\beta_{j}\right) \cdot t+i \cdot \operatorname{Im}\left(\beta_{j}\right) \cdot t\right) .
\end{aligned}
$$

For stability we require that $\operatorname{Re}\left(\beta_{j}\right) \leqslant 0 \quad(j=0,1)$. From (3.18) we then see that this can only be achieved if $\hat{A}$ is real and negative.

As mentioned before, $A \phi$ is the discrete approximation of $\partial \phi / \partial n$, and is obtained from the integral equation (2.9). Denoting $\partial \phi / \partial n$ by $\psi$ for convenience, the integral equation for $\mathbf{X}=\mathbf{x}_{m n}$ is

$$
\begin{aligned}
\phi\left(\mathbf{x}_{m n}\right) & =-\frac{1}{2 \pi} \int_{-\infty}^{\infty} \int_{-\infty}^{\infty} \psi \frac{1}{r} d \xi d \vartheta \\
& =K I N V(\psi)\left(\mathbf{x}_{m n}\right)
\end{aligned}
$$


or

$$
\psi\left(\mathbf{x}_{m n}\right)=K I N V^{-1}(\phi)\left(\mathbf{x}_{m n}\right)
$$

assuming that $K I N V^{-1}$ exists. tion

Discretizing this equation gives the discrete approxima-

$$
\psi=A \phi
$$

Fourier transformation gives

$$
\hat{\psi}\left(k_{x}, k_{y}\right)=\hat{A}\left(k_{x}, k_{y}\right) \hat{\phi}\left(k_{x}, k_{y}\right) .
$$

The Fourier symbol $\hat{A}$ of $A$ can easily be found from

$$
\begin{gathered}
\hat{A}=A\left(\exp \left(i k_{x} x+i k_{y} y\right)\right)\left(\mathbf{x}_{m n}\right) / \exp \left(i k_{x} x_{m}+i k_{y} y_{n}\right), \\
k_{x}, k_{y} \text { real. }
\end{gathered}
$$

For the lowest order consistent approximation of the integral equation, $\phi$ is a linear function and $\psi$ a constant function over each panel. With panel size $h=\Delta x=\Delta y$, the approximation can be written as

$$
\begin{aligned}
\phi\left(\mathbf{x}_{m n}\right) & =-\frac{1}{2 \pi} \sum_{i=-\infty}^{\infty} \sum_{j=-\infty}^{\infty} \int_{x_{i}-h / 2}^{x_{i}+h / 2} \int_{y_{i}-h / 2}^{y_{i}+h / 2} \psi\left(\mathbf{x}_{i j}\right) \frac{1}{r} d \xi d \vartheta \\
& =-\frac{1}{2 \pi} \sum_{p=-\infty}^{\infty} \sum_{q=-\infty}^{\infty}\left[b_{p q} \cdot \psi_{m-p, n-q}\right] \\
& =\operatorname{AINV}(\psi)\left(\mathbf{x}_{m n}\right)
\end{aligned}
$$

where $p=m-i$ and $q=n-j$ is substituted. The coefficients

$b_{p q}=b_{m-i, n-j}$

$$
=\int_{x_{i}-h / 2}^{x_{i}+h / 2} \int_{y_{i}-h / 2}^{y_{i}+h / 2} \frac{1}{\sqrt{\left(x_{m}-\xi\right)^{2}+\left(y_{n}-\vartheta\right)^{2}}} d \xi d \vartheta
$$

are positive and symmetric in $p$ and $q: b_{p q}=b_{-p,-q}=$ $b_{-p, q}=b_{p,-q}$. The Fourier symbol of $A I N V$ is

$$
\begin{aligned}
\hat{A} I N V= & A I N V\left(\exp \left(i k_{x} x+i k_{y} y\right)\right)\left(\mathbf{x}_{m n}\right) / \exp \left(i k_{x} x_{m}+i k_{y} y_{n}\right) \\
= & -\frac{1}{2 \pi} \sum_{p=-\infty}^{\infty} \sum_{q=-\infty}^{\infty}\left[b_{p q} \cdot \exp \left(i k_{x} x_{-p}+i k_{y} y_{-q}\right)\right] \\
= & -\frac{1}{2 \pi} \sum_{p=-\infty}^{\infty}\left[b_{p 0}+\sum_{q=1}^{\infty} b_{p q} \cdot 2 \cdot \cos \left(k_{y} q h\right)\right. \\
& \left.\times \exp \left(-i k_{x} p h\right)\right]
\end{aligned}
$$

$$
\begin{aligned}
= & -\frac{1}{2 \pi}\left[b_{00}+\sum_{q=1}^{\infty} b_{0 q} \cdot 2 \cdot \cos \left(k_{y} q h\right)\right. \\
& +\sum_{p=1}^{\infty} b_{p 0} \cdot 2 \cdot \cos \left(k_{x} p h\right) \\
& \left.+\sum_{p=1}^{\infty} \sum_{q=1}^{\infty} b_{p q} \cdot 4 \cdot \cos \left(k_{x} p h\right) \cdot \cos \left(k_{y} q h\right)\right]
\end{aligned}
$$

and

$$
\hat{A}(\mathbf{k})=\hat{A} I N V^{-1}(\mathbf{k})
$$

Since $\hat{K} I N V^{-1}=\hat{\partial} / \partial n=-\sqrt{k_{x}^{2}+k_{y}^{2}}$, the operator $\hat{A} I N V^{-1}=\hat{A}$ must be an approximation of $-k$, and, for stability, it must be real.

Now, $\operatorname{Im}(\hat{A})=0$ follows directly from the expression (3.27), wich is real. $\operatorname{Re}(\hat{A}) \leqslant 0$ can be seen from the following. The coefficients $b_{p q}$ are all positive and decrease as $1 / p$ and $1 / q$ for $p \rightarrow \infty$ and $q \rightarrow \infty$, respectively. Since

$$
\sum_{p=1}^{\infty} \frac{\cos (p x)}{p}=-\log \left(2 \cdot \sin \left(\frac{x}{2}\right)\right), \quad 0<x<2 \pi,
$$

is positive, the series in (3.27) converge to a negative real value for all $k_{x}, k_{y} \neq 0$.

Figure 2 shows $-\boldsymbol{h} \cdot \hat{A}(\mathbf{k})$ as a function of $h k$ when $k_{x}=k_{y}$. Clearly the scheme gives a very good approximation of $k$, meaning that there is very little numerical dispersion (the scheme is not dissipative due to $\operatorname{Re}\left(\beta_{j}\right)=0$ ).

We have shown that $\hat{A}(\mathbf{k})$ is real and negative, so we have

THEOREM 3.2. The semi-discrete approximation of the free surface problem without boundaries satisfies the Von Neumann stability condition.

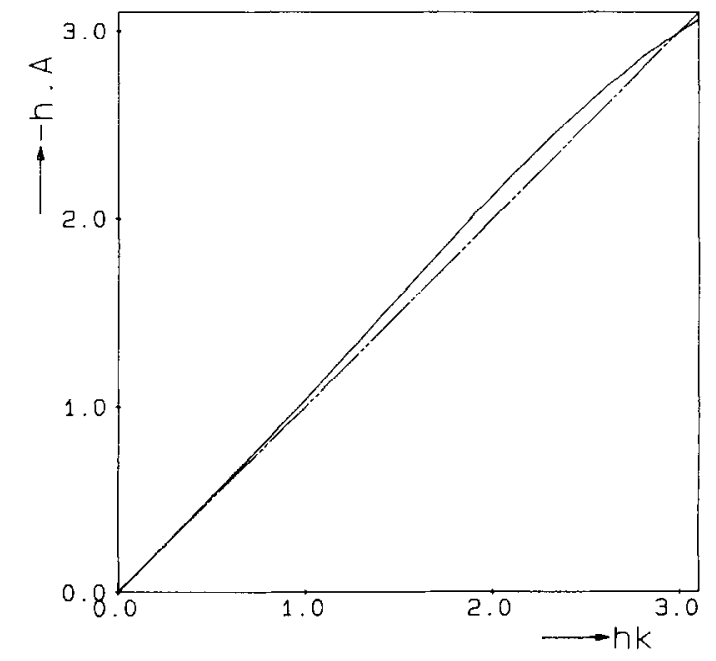

FIG. 2. The symbol $-h A$ compared to $h k$ as a function of $h k$ on $(0, \pi)$ for $k_{x}=k_{y}$. 
We will therefore assume that the discrete model is Cauchy stable.

\subsection{Stability of the Half-Space Problem}

Now consider the half-infinite free surface domain $x \leqslant 0$ by introducing a vertical boundary at $x=0$. For stability we require that no solutions of the form (3.13) exist that violate the NMC.

We will start with the conditions (3.9) and (3.10): assume that $\left|F_{x}\right| \leqslant 1$ and $|G|>1$, i.e., solutions which are bounded in space and with $\operatorname{Re}(\beta)>0$. Here we must admit solutions with $\operatorname{Im}\left(k_{x}\right)=0$, as for Cauchy stability, and with $\operatorname{Im}\left(k_{x}\right)<0$, since these solutions are in $l_{2}$. Thus we have to consider the case $\operatorname{Im}\left(k_{x}\right) \leqslant 0$. For the same reason we must have $\operatorname{Im}\left(k_{z}\right) \leqslant 0\left(\left|F_{z}\right| \leqslant 1\right)$, and we maintain $\operatorname{Im}\left(k_{y}\right)=0$.

In order to prove these first two conditions for the discretizations used here, an assumption must be made concerning the approximation of the normal derivative. Insertion of the normal mode (3.13) in the integral equation again gives the symbol $\hat{A}(\mathbf{k})$ of the discrete operator $A$, which approximates the normal derivative $\partial / \partial n$ on the vertical boundaries. We make the

\section{Proposition 3.1. The symbol}

$$
\begin{aligned}
\hat{A}\left(k_{x}, k_{y}, k_{z}\right)= & A\left(\exp \left(i k_{x} x+i k_{y} y+i k_{z} z\right)\right)\left(\mathbf{x}_{m n l}\right) \\
& / \exp \left(i k_{x} x_{m}+i k_{y} y_{n}+i k_{z} z_{l}\right),
\end{aligned}
$$

with $\operatorname{Im}\left(k_{x}\right) \leqslant 0, \operatorname{Im}\left(k_{y}\right)=0$, and $\operatorname{Im}\left(k_{z}\right) \leqslant 0$, satisfies on the boundary at $x=0$ of $\Omega=\{\mathbf{x} \mid x \leqslant 0, y \in \mathbb{R}, z \leqslant 0\}$ the condition:

$$
\operatorname{Re}\left(\hat{A}\left(k_{x}, k_{y}, k_{z}\right)\right) \leqslant 0 .
$$

Above we have seen that the proposition holds for the infinite plane $z=0$ of the half-space $\{\mathbf{x} \mid x \in \mathbb{R}, y \in \mathbb{R}, z \leqslant 0\}$ with $\operatorname{Im}\left(k_{x}\right)=0$, since the symbol $\hat{A}$ is independent of $k_{z}$ in that case. The same conclusions can be made for the boundary $x=0$ of the half-space $\{\mathbf{x} \mid x \leqslant 0, y \in \mathbb{R}, z \in \mathbb{R}\}$ with $\operatorname{Im}\left(k_{y}\right)=0$ and $\operatorname{Im}\left(k_{z}\right)=0$. It cannot be proved easily for the boundaries of the quarter-space of Proposition 3.1 with $\operatorname{Im}\left(k_{x}\right) \leqslant 0$ and $\operatorname{Im}\left(k_{z}\right) \leqslant 0$.

However, the proposition is supported by the fact that the eigenvalues of the part in the matrix $-C_{s}^{-1} C_{d}$ (where $C_{s}$ contains the source coefficients of the matrix determined for the panel method and $C_{d}$ contains the dipole coefficients) corresponding to one boundary were found to have no positive real part for the test geometries described in the following section, or for any other geometry used so far. Hence (3.30) is assumed to be true for solutions on the halfplane.

Now it is easy to show that Eq. (3.9) and (3.10) are satisfied. For this, assume that (3.13), with $\operatorname{Im}\left(k_{x}\right) \leqslant 0$,
$\operatorname{Im}\left(k_{y}\right)=0$, and $\operatorname{Im}\left(k_{z}\right) \leqslant 0$, is a solution of the free surface equations, and $\operatorname{Re}(\beta)>0$. Now the scheme is stable only if this solution does not satisfy the chosen boundary condition in $x=0$.

The first-order absorbing boundary condition (2.5) can be written as

$$
\frac{\partial \phi}{\partial t}=\frac{c}{\cos (\alpha)} \frac{\partial \phi}{\partial n}, \quad|\alpha|<\pi / 2
$$

Inserting (3.13) gives

$$
\beta \cdot \hat{\phi}=\frac{c}{\cos (\alpha)} \cdot v \cdot \hat{\phi}, \quad \text { with } \quad v=\hat{A}
$$

No solution with $\operatorname{Re}(\beta)>0$ can exist, since $\operatorname{Re}(v) \leqslant 0$ according to (3.30). Hence this discretization is stable in the sense of Godunov-Ryabenkii (note that (3.30) also guarantees the internal Gauchy stability of the scheme on the artificial boundary).

By using $Y=a \cdot(\partial \phi / \partial t)-b \cdot(\partial \phi / \partial n)$, the second-order absorbing boundary condition (2.6) can be written as

$$
\begin{aligned}
& \frac{\partial \phi}{\partial t}=\frac{b}{a} \cdot \frac{\partial \phi}{\partial n}+\frac{1}{a} \cdot Y, \\
& \frac{\partial Y}{\partial t}=c^{2} \cdot \frac{\partial^{2} \phi}{\partial s^{2}}-e \cdot \phi
\end{aligned}
$$

where $s$ is the horizontal tangential coordinate to the boundary (see Fig. 1).

Because in this paper only problems with a horizontal bottom will be considered, a regular panel distribution can be chosen on the vertical boundaries, so that the horizontal tangential derivatives are approximated by a finite diffcrence approximation on a molecule of three equidistant collocation points.

Inserting (3.13) in (3.33) and assuming $\operatorname{Re}(\hat{A})=$ $\operatorname{Re}(v) \leqslant 0$, again results in eigenvalues $\beta$ with $\operatorname{Re}(\beta) \leqslant 0$, so that the discretization of (3.33) is stable. Again, the assumption $\operatorname{Re}(v) \leqslant 0$ also guarantees the internal stability of the discretization on $x=0$. Therefore we have

THEOREM 3.3. Assuming (3.30) to hold, the numerical schemes for the free surface problem with boundary in $x=0$ and first-or second-order absorbing boundary conditions imposed thereon, are stable in the sense of GodunovRyabenkii; i.e., no solutions exist with $\left|F_{x}\right| \leqslant 1$ and $|G|>1$.

Now consider the case $\left|F_{x}\right| \leqslant 1$ and $|G|=1$. Assuming that (3.13) is a solution of the free surface equations, it is inserted again in the boundary conditions. From (3.32) it is clear that no solutions exist with $\left|F_{x}\right|<1$ and $|G|=1(\operatorname{Re}(\beta)=0)$ for the first-order absorbing boundary 
condition. Also, for any perturbed $\beta$ with $\operatorname{Re}(\beta)>0$, we have $\operatorname{Re}(v)>0$, so that conditions (3.11) and (3.12) are satisfied.

For the second-order absorbing boundary condition we have the transformed equation

$$
\beta\left[\begin{array}{l}
\hat{\phi} \\
\hat{Y}
\end{array}\right]=\left[\begin{array}{cc}
\frac{b}{a} \cdot v & \frac{1}{a} \\
-c^{2} \mu-e & 0
\end{array}\right]\left[\begin{array}{l}
\hat{\phi} \\
\hat{Y}
\end{array}\right],
$$

with $\operatorname{Re}(v) \leqslant 0$, and $\mu$ stems from the central discretization of the second derivative $\partial^{2} \phi / \partial s^{2}$ :

$$
\begin{aligned}
\mu & =-\frac{\exp \left(i k_{y} h\right)-2+\exp \left(-i k_{y} h\right)}{h^{2}} \\
& =\frac{2-2 \cdot \cos \left(k_{y} h\right)}{h^{2}} .
\end{aligned}
$$

Hence $\mu$ is real and $\mu \geqslant 0$.

The eigenvalues $\beta_{i}$ of the matrix in Eq. (3.34) satisfy

$$
\beta_{i}^{2}-\left(\frac{b}{a} \cdot v\right) \cdot \beta_{i}+\frac{c^{2} \cdot \mu+e}{a}=0
$$

Inserting a perturbed eigensolution $\beta_{i}=\delta+i \varepsilon$ with $\delta>0$ in (3.36) gives

$$
\begin{gathered}
\delta^{2}-\varepsilon^{2}+i \cdot 2 \cdot \delta \cdot \varepsilon-\frac{b}{a} \cdot v \cdot(\delta+i \cdot \varepsilon) \\
+\frac{c^{2} \cdot \mu+e}{a}=0,
\end{gathered}
$$

from which we derive that

$$
\begin{aligned}
\operatorname{Re}(v)= & \frac{a}{b} \cdot \delta \cdot\left(\delta^{2}+\varepsilon^{2}+\frac{c^{2} \cdot \mu+e}{a}\right) \\
& \times\left(\delta^{2}+\varepsilon^{2}\right)^{-1}>0 .
\end{aligned}
$$

Thus no solutions exist with $\left|F_{x}\right| \leqslant 1$ and $|G|=1$ that violate the conditions (3.11)-(3.12). We have shown

THEOREM 3.4. The numerical schemes for the semidiscrete free surface problem, with a boundary in $x=0$ and the first - or second-order absorbing boundary condition imposed on that boundary, satisfy the NMC, if (3.30) is assumed.

With these stability results for the half-infinite domain, we now turn to the corner problem; i.e., we will investigate the stability of the scheme if a corner is present.

\subsection{Stability of the Quarter-Space Problem}

In practical calculations the fluid domain is truncated in all horizontal directions by (artificial) boundaries, introducing corners and vertical edges in the domain. Consider, therefore, the domain $\Omega=\{\mathbf{x} \mid x \leqslant 0, y \leqslant 0$, $z \leqslant 0\}$, with boundaries at $x=0$ and $y=0$, and suppose the boundary conditions imposed on these boundaries are $B_{x}=0$ and $B_{y}=0$, respectively.

Again we consider solutions of the form (3.13) and require that no such solution can exist that violates the NMC. For the half-space problem, solutions were admitted with $\operatorname{Im}\left(k_{x}\right) \leqslant 0, \operatorname{Im}\left(k_{y}\right)=0$, and $\operatorname{Im}\left(k_{z}\right) \leqslant 0$. Here solutions with $\operatorname{Im}\left(k_{x}\right) \leqslant 0, \operatorname{Im}\left(k_{y}\right) \leqslant 0$, and $\operatorname{Im}\left(k_{z}\right) \leqslant 0$ must be investigated. Therefore, we need an extension of Proposition 3.1, to be able to make further investigations:

Proposition 3.2. The symbol

$$
\begin{aligned}
\hat{A}\left(k_{x}, k_{y}, k_{z}\right)= & A\left(\exp \left(i k_{x} x+i k_{y} y+i k_{z} z\right)\right)\left(\mathbf{x}_{m n l}\right) \\
& / \exp \left(i k_{x} x_{m}+i k_{y} y_{n}+i k_{z} z_{l}\right),
\end{aligned}
$$

with $\operatorname{Im}\left(k_{x}\right) \leqslant 0, \operatorname{Im}\left(k_{y}\right) \leqslant 0$, and $\operatorname{Im}\left(k_{z}\right) \leqslant 0$, satisfies on the boundaries at $x=0$ and at $y=0$ of $\Omega=\{\mathbf{x} \mid x \leqslant 0, y \leqslant 0$, $z \leqslant 0\}$ the condition:

$$
\operatorname{Re}\left(\hat{A}\left(k_{x}, k_{y}, k_{z}\right)\right) \leqslant 0 .
$$

This proposition is supported by the same arguments as Proposition 3.1.

Consider the boundary at $x=0$ (of course, the analysis is also applicable to $y=0$ ). The collocation points on the boundary are $\mathbf{x}_{m n l}=(0,-(n-1 / 2) \cdot h,-(l-1 / 2) \cdot h)$, $n=1,2, \ldots ; l=1,2, \ldots$.

In the internal points $n=2,3, \ldots$, the same boundary condition is imposed as in the half-space case. In $n=1$, however, this is not always possible. No special treatment is required for the first-order absorbing boundary condition, but in the second-order case a discretization of (2.7) is needed in $n=1$.

So we assume that a solution (3.13) satisfies the free surface equations, the boundary condition on $y=0$, and the boundary condition on $x=0$ in the interior points $n=2,3, \ldots$, satisfying any of the four cases of the NMC, with $|F| \leqslant 1$ and $|G| \geqslant 1$. Then the scheme is stable if this solution is excluded by the boundary condition in the end point $n=1$.

First consider the cases (3.9)-(3.10): $\left|F_{x}\right| \leqslant 1,\left|F_{y}\right| \leqslant 1$, $\left|F_{z}\right| \leqslant 1$, and $|G|>1(\operatorname{Re}(\beta)>0)$. As in (3.32), the first-order absorbing boundary condition gives

$$
\beta \cdot \hat{\phi}=\frac{c}{\cos (\alpha)} \cdot v \cdot \hat{\phi}, \quad \text { with } \quad v=\hat{A}
$$


No solutions can exist with $\operatorname{Re}(\beta)>0$, since $\operatorname{Re}(v) \leqslant 0$ according to Eq. (3.39).

The second-order absorbing boundary condition (2.7) in $n=1$ is rewritten as a system with first time derivatives and no spatial derivatives of the variable $Z$, which "exists" only in $n=1$ :

$$
\begin{aligned}
& \frac{\partial \phi}{\partial t}=-\frac{c}{\sin \left(\alpha_{i}\right)} \cdot \frac{\partial \phi}{\partial s}+\frac{c}{\cos \left(\alpha_{2}\right)} \cdot \frac{\partial \phi}{\partial n}+Z, \\
& \frac{\partial Z}{\partial t}=\frac{c^{2}}{\sin \left(\alpha_{1}\right) \cdot \cos \left(\alpha_{2}\right)} \cdot \frac{\partial^{2} \phi}{\partial s \partial n} .
\end{aligned}
$$

The tangential derivative $\partial \phi / \partial s$ is approximated using a one-sided finite difference scheme, such that the approximation of $\partial \hat{\phi} / \partial s$ in $n=1$ can be written as $\rho \cdot \hat{\phi}$, with $\operatorname{Re}(\rho) \geqslant 0$. In the computations a three-point second-order scheme was chosen,

$$
\left.\frac{\widetilde{\partial} \phi}{\partial s}\right|_{n=1}=\frac{3 \cdot \phi_{1}-4 \cdot \phi_{2}+\phi_{3}}{2 \cdot h}
$$

with $\phi_{i}=\phi_{y=-(i-1 / 2) h}$.

Inserting the elementary solution (3.13) gives $\rho$ :

$$
\begin{aligned}
\rho & =\frac{1}{2 h}\left(3-4 \cdot \exp \left(-i k_{y} h\right)+\exp \left(-2 \cdot i k_{y} h\right)\right) \\
& \left.=\frac{1}{2 h}\left(\left(\exp \left(-i k_{y} h\right)\right)-2\right)^{2}-1\right) .
\end{aligned}
$$

From this equation it follows that $\left(\operatorname{Im}\left(k_{y}\right) \leqslant 0\right.$ !):

$$
\begin{aligned}
\operatorname{Re}(\rho)= & \frac{1}{2 h}\left(\left(\exp \left(\operatorname{Im}\left(k_{y}\right) h\right) \cdot \cos \left(\operatorname{Re}\left(k_{y}\right) h\right)-2\right)^{2}\right. \\
& \left.-\left(\exp \left(\operatorname{Im}\left(k_{y}\right) h\right) \cdot \sin \left(\operatorname{Re}\left(k_{y}\right) h\right)\right)^{2}-1\right) \\
= & \frac{1}{2 h}\left(2 \cdot\left(\exp \left(\operatorname{Im}\left(k_{y}\right) h\right) \cdot \cos \left(\operatorname{Re}\left(k_{y}\right) h\right)-1\right)^{2}\right. \\
& \left.-\left(\exp \left(\operatorname{Im}\left(k_{y}\right) h\right)\right)^{2}+1\right) .
\end{aligned}
$$

Clearly, $\operatorname{Re}(\rho) \geqslant 0$ for all $k_{y} \cdot \operatorname{Re}(\rho)=0$ only occurs when $\operatorname{Re}\left(k_{y}\right)=\operatorname{Re}\left(k_{x}\right)=0$. Now inserting (3.13) into the absorbing boundary condition (3.41) in $n=1$ gives

$$
\beta\left[\begin{array}{c}
\hat{\phi} \\
\hat{Z}
\end{array}\right]=\left[\begin{array}{cc}
-\frac{c}{\sin \left(\alpha_{1}\right)} \cdot \rho+\frac{c}{\cos \left(\alpha_{2}\right)} \cdot v & 1 \\
\frac{c^{2}}{\sin \left(\alpha_{1}\right) \cdot \cos \left(\alpha_{2}\right)} \cdot \rho \cdot v & 0
\end{array}\right]\left[\begin{array}{l}
\hat{\phi} \\
\hat{Z}
\end{array}\right]
$$

The eigenvalues $\beta_{i}$ of the matrix are $\left(-c / \sin \left(\alpha_{1}\right)\right) \cdot \rho$ and $\left(c / \cos \left(\alpha_{2}\right)\right) \cdot v$. No solutions with $\operatorname{Re}(\beta)>0$ satisfy $(3.45)$, since $\operatorname{Re}(v) \leqslant 0$ and $\operatorname{Re}(\rho) \geqslant 0$; hence, this stability criterion is satisfied.

THEOREM 3.5. Assuming (3.39) holds, the numerical schemes for the free surface problem with boundary in $x=0$ and $y=0$, and the described first - or second-order absorbing boundary conditions imposed thereon, are stable in the sense of Godunov-Ryabenkii; i.e., no solutions exist with $\left|F_{x}\right| \leqslant 1$, $\left|F_{y}\right| \leqslant 1$, and $|G|>1$.

In a similar way as before, it is easily seen that no solutions with $\left|F_{x}\right| \leqslant 1,\left|F_{y}\right| \leqslant 1$, and $|G|=1$ can exist for either of the two boundary conditions. Hence we have, assuming that on $y=0$ an appropriate boundary condition is chosen, as well:

THEOREM 3.6. The numerical schemes for the semidiscrete free surface problem, with a boundary in $x=0$ and $y=0$, and the first - or second-order absorbing boundary condition imposed on those boundaries, satisfy the NMC, if (3.39) is assumed.

Theorem 3.6 provides a strong (and necessary) argument for stability of the scheme, but as mentioned, it is not sufficient. Polynomial growth may still be possible, and also we have considered only homogeneous boundary conditions. Forcing terms in the boundary conditions to simulate incoming waves may destabilize the scheme.

\subsection{Conclusions}

We can conclude that the absorbing boundary conditions satisfy strong and necessary conditions for stability, under the assumption that Propositions 3.1 and 3.2 hold. Arguments have been given that support these propositions.

\section{NUMERICAL RESULTS}

In this section the conclusions about the stability of the proposed boundary conditions will be tested by implementing them in the panel method. For specific choices of the parameters their reflection properties will be checked.

\subsection{Test Geometries}

In order to test the boundary conditions, two different geometries are used. The boundary conditions have been developed for plane waves. Their behaviour can be tested by using a model of a rectangular wave tank. The tank has a length of $40 \mathrm{~m}$, its width is $30 \mathrm{~m}$, and its depth is $6 \mathrm{~m}$ (see Fig. 3).

The second model is a less trivial model. It is used to simulate diffracting waves on a cylinder (see Fig. 4). The plane $y=0$ and the bottom are planes of symmetry. The program used to solve the problem (see [8]) has the 


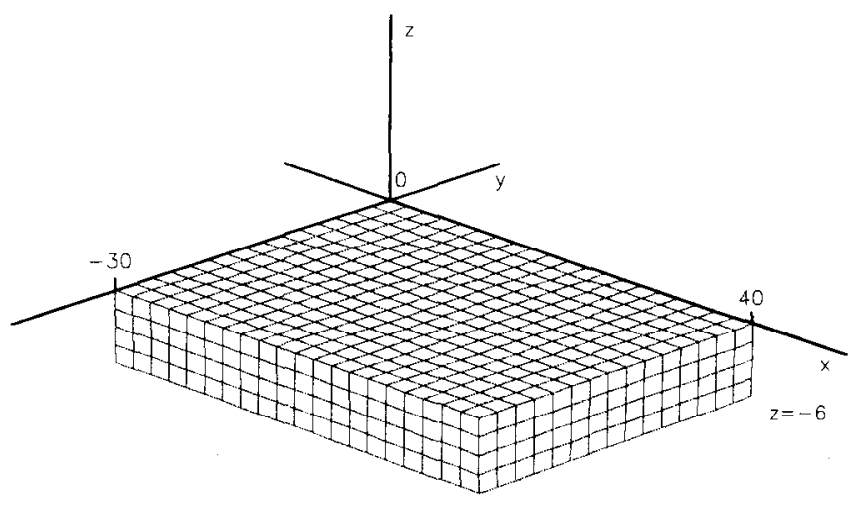

FIG. 3. Grid on the rectangular wave tank.

possibility to include these symmetries, so that only a part of the problem has to be solved.

Waves can be generated from a (vertical) boundary of a model by using a first-order absorbing boundary condition with a generating function $f$ :

$$
\frac{\partial \phi}{\partial t}=\frac{c}{\cos (\alpha)} \cdot \frac{\partial \phi}{\partial n}+f
$$

Plane waves can be generated in the first model by imposing Eq. (4.1) on one or two vertical boundaries (the boundaries where the waves enter the model), while the wall condition or first- or second-order absorbing boundary conditions can be imposed on the other boundaries. Equation (4.1) can also be used to generate waves in the second model, from the boundary at $x=0$. This condition is first-order absorbing, so that waves that are reflected by the pile can be absorbed. On the other artificial boundaries, absorbing boundary conditions can be imposed, so that the incoming and the diffracted waves are absorbed.

In Fig. 5 a horizontal section of both models is given. In the first model the boundaries are numbered for future reference.

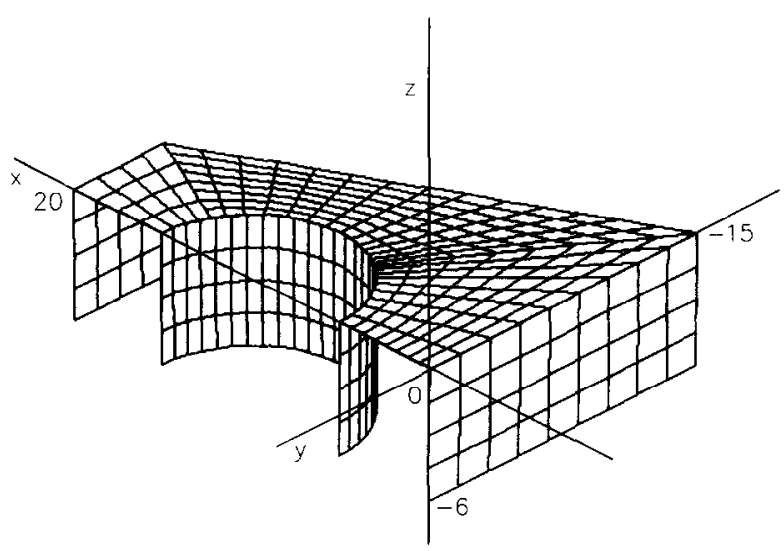

FIG. 4. Model for simulating diffracting waves on a cylinder.
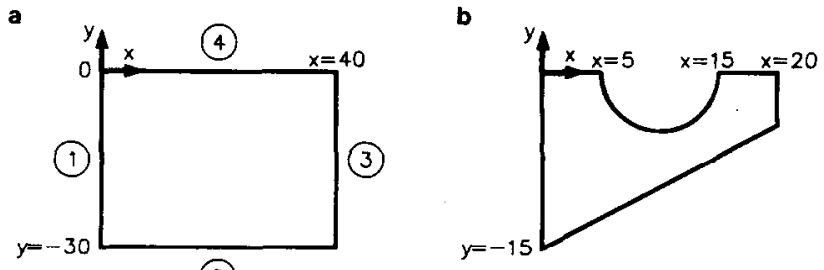

(2)

FIG. 5. (a) Surface of the first test model. (b) Surface of the second test model.

\subsection{Results on the Rectangular Wave Tank}

In this subsection the stability and reflection properties of the absorbing boundary conditions will be tested for plane waves, using the rectangular wave tank.

Waves propagating in different directions, say at an angle $\beta(0 \leqslant \beta \leqslant \pi / 2)$ with the $x$-axis, can be simulated by using the following harmonic solution of the free surface problem as the initial solution at $t=0$ :

$$
\begin{aligned}
\phi(x, y, z, t)= & A \frac{\omega}{k} \frac{\cosh (k \cdot(z+h))}{\sinh (k \cdot h)} \\
& \times \cos (k(x \cdot \cos (\beta)+y \cdot \sin (\beta))-\omega \cdot t), \\
\eta(x, y, t)= & -A \sin (k(x \cdot \cos (\beta)+y \cdot \sin (\beta))-\omega \cdot t) \\
& (z=0) .
\end{aligned}
$$

In this expression $A$ stands for the amplitude of the wave, $\omega$ is the angular frequency, and $k$ is the wave number. In the tests these parameters will have the following values:

amplitude of the incoming wave: $\quad A=1 \mathrm{~m}$

angular frequency:

$\omega=1 \mathrm{rad} / \mathrm{s}$

wave number:

$k=0.145208 \mathrm{rad} / \mathrm{m}$.

The time steps are chosen small enough, so that no instabilities develop due to the Runge-Kutta time integration method.

The first-order conditions (Eq. (3.31) or (4.1)) are imposed on boundaries 1,2 , and 4 , such that they are exact for the waves to be simulated. On boundary 3 first- or second-order absorbing boundary conditions will be tested.

In order to obtain confirmation on the stability of the various boundary conditions, four numerical exeriments are done, with an incoming wave at an angle of incidence $\theta=\pi / 6$ (see Fig. 1). Table I summarizes the conclusions from the previous section on the stability of the various choices of boundary conditions. The values of the parameters shown in this table are chosen such that the incident wave should be perfectly absorbed. Any error in the 
TABLE I

Theoretical Stability Results

\begin{tabular}{llccc}
\hline $\begin{array}{c}\text { Test } \\
\text { number }\end{array}$ & $\begin{array}{c}\text { Boundary } \\
\text { condition }\end{array}$ & Order & $\begin{array}{c}\text { Angles of best } \\
\text { absorption }\end{array}$ & Stable \\
\hline 1 & 1st & $\alpha=\frac{\pi}{6}$ & Yes \\
2 & 2nd & $\alpha_{1}=\frac{\pi}{6}, \alpha_{2}=0$ & Yes \\
& (3.33) & 1st & & \\
3 & (3.33) & 2nd & $\alpha_{1}=\frac{\pi}{6}, \alpha_{2}=0$ & Yes \\
& End points: (3.41) & 2nd & & \\
4 & (3.33) & 2nd & $\alpha_{1}=\frac{\pi}{6}, \alpha_{2}=0$ & No \\
& End points: (3.33) & 2nd & &
\end{tabular}

computations hence must be due to the discretizations or, worse, may be caused by unstable behaviour.

In all cases the experiments confirm the theoretical results of Table I.

The errors that occur in the first three cases are almost equal, because these are due to the discretizations, viz. the numerical dispersion of the scheme at the free surface, and are not caused by the boundary conditions. After $7.5 \mathrm{~s}$ (which is longer than one period) the absolute errors in $\eta$ are like those in Fig. 6. After a longer time, the magnitude of the errors does not increase for these boundary conditions.

Imposing the second-order condition (3.33) in all collocation points of a boundary (test 4) yields an unstable system, as predicted by the theory. The instabilities develop in the begin and end points of the sccond-order boundary. This

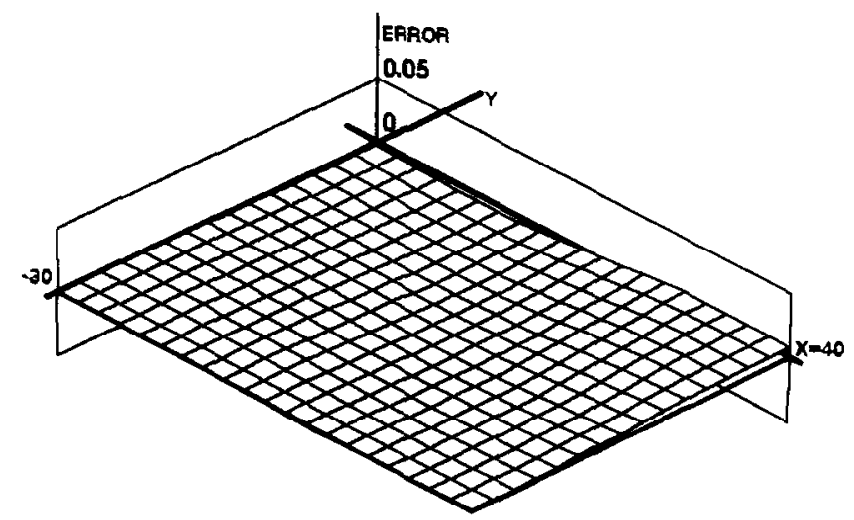

FIG. 6. The errors in the elevation on the free surface of the first three tests.

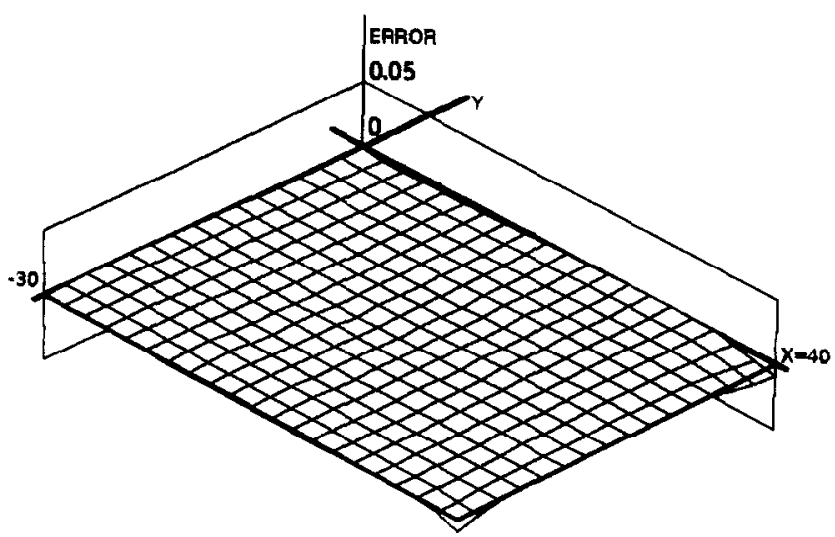

FIG. 7. The errors in the elevation in the fourth test after $1.5 \mathrm{~s}$.

can be concluded most easily from the results after a short period. The absolute errors after $1.55 \mathrm{~s}$ for the fourth test are therefore shown in Fig. 7. The errors propagate over the boundaries, but they keep growing for $t>1.5 \mathrm{~s}$.

With respect to the reflection properties of the boundary conditions in the first three tests, no reflected wave can be distinguished in the numerical results, confirming that the waves should be completely absorbed.

Next we will consider some cases in which, theoretically, reflections should occur. For these tests the same boundary conditions are used as in the previous test, but the incident wave is chosen to come from a different direction $(\theta=\pi / 4)$.

Results of the test cases in Table II are presented in Figs 8-10. These figures show the resulting errors on the free surface after $3 \mathrm{~s}$. Also figures are added that give the development of the errors in time in the point $(x, y, z)=(39,-3,0)$, which lies on the free surface, close to the corner.

TABLE II

Theoretical Reflection Coefficients

\begin{tabular}{ccccc}
\hline $\begin{array}{c}\text { Test } \\
\text { number }\end{array}$ & $\begin{array}{c}\text { Boundary } \\
\text { condition }\end{array}$ & Order & $\begin{array}{c}\text { Angles of best } \\
\text { absorption }\end{array}$ & $\begin{array}{c}\text { Reflection } \\
\text { coefficients }\end{array}$ \\
\hline 5 & $(3.31)$ & 1 st & $\alpha=\frac{\pi}{6}$ & -0.101 \\
6 & $(3.33)$ & 2nd & $\alpha_{1}=\frac{\pi}{6}, \alpha_{2}=0$ & -0.017 \\
& End points: $(3.31)$ & 1st & & -0.101 \\
7 & (3.33) & 2nd & $\alpha_{1}-\frac{\pi}{6}, \alpha_{2}=0$ & -0.017 \\
& End points: $(3.41)$ & 2nd & & \\
\hline
\end{tabular}



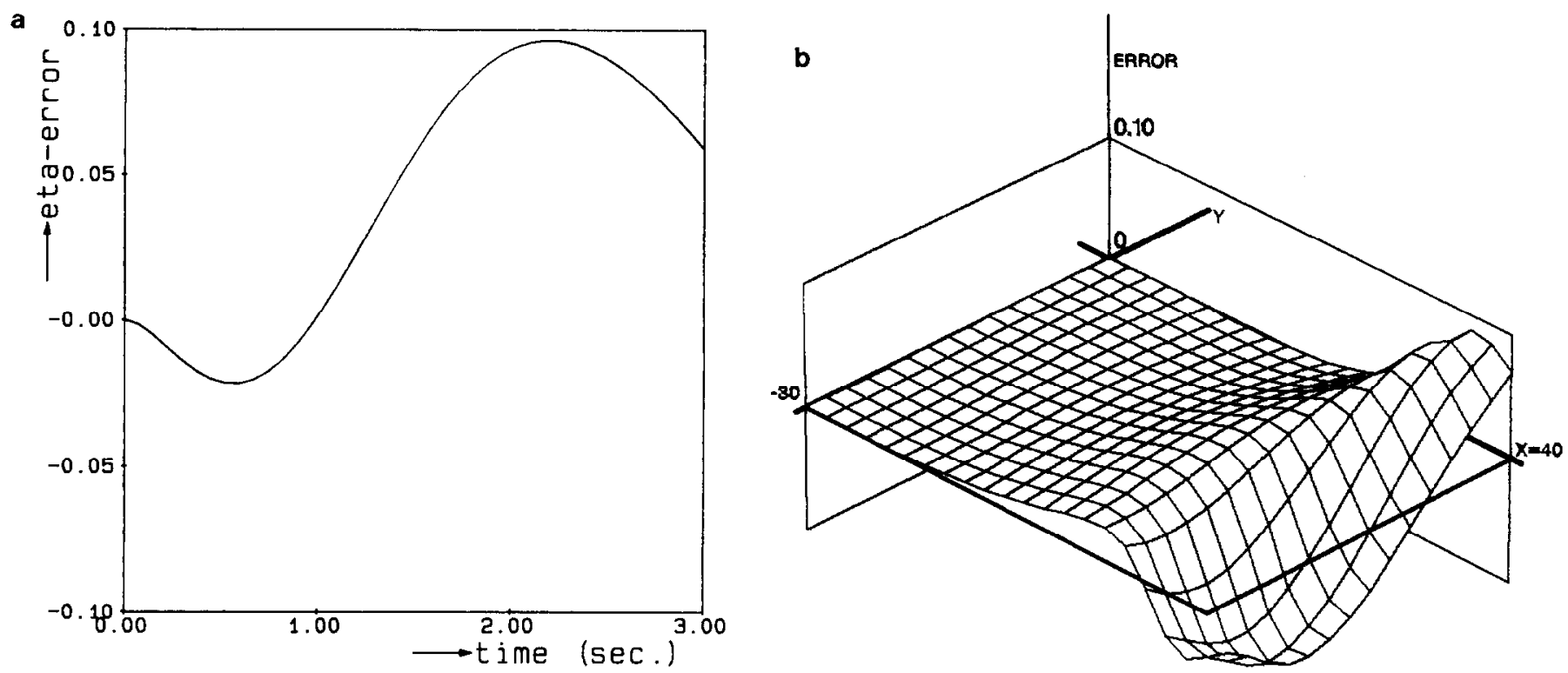

FIG. 8. (a) Eta error in time. Time development of the error in test $5,(x, y, z)=(39,-3,0)$. (b) Errors on the free surface in test 5 after $3 \mathrm{~s}$ due to reflections.

Figures 8-10 clearly show that the errors in these tests are dominated by the reflected wave, but they are not larger than theoretically expected. The reflections that appear have an amplitude which is of the same magnitude as the reflection coefficient (notice that the incoming wave has an amplitude $A=1$ ). It is also clear that the reflections are reduced by using higher order absorbing boundary conditions, even though the angles $\alpha_{1}$ and $\alpha_{2}$ are not directed toward the incoming wave. Using second-order conditions with first-order conditions in the beginning and ending collocation points provides high reflections in the corners, as expected.

\subsection{Results on Diffracted Waves}

In this subsection, we will test how these conditions absorb waves which are diffracted by a circular pile. The incoming waves are the same as those used in the previous

a
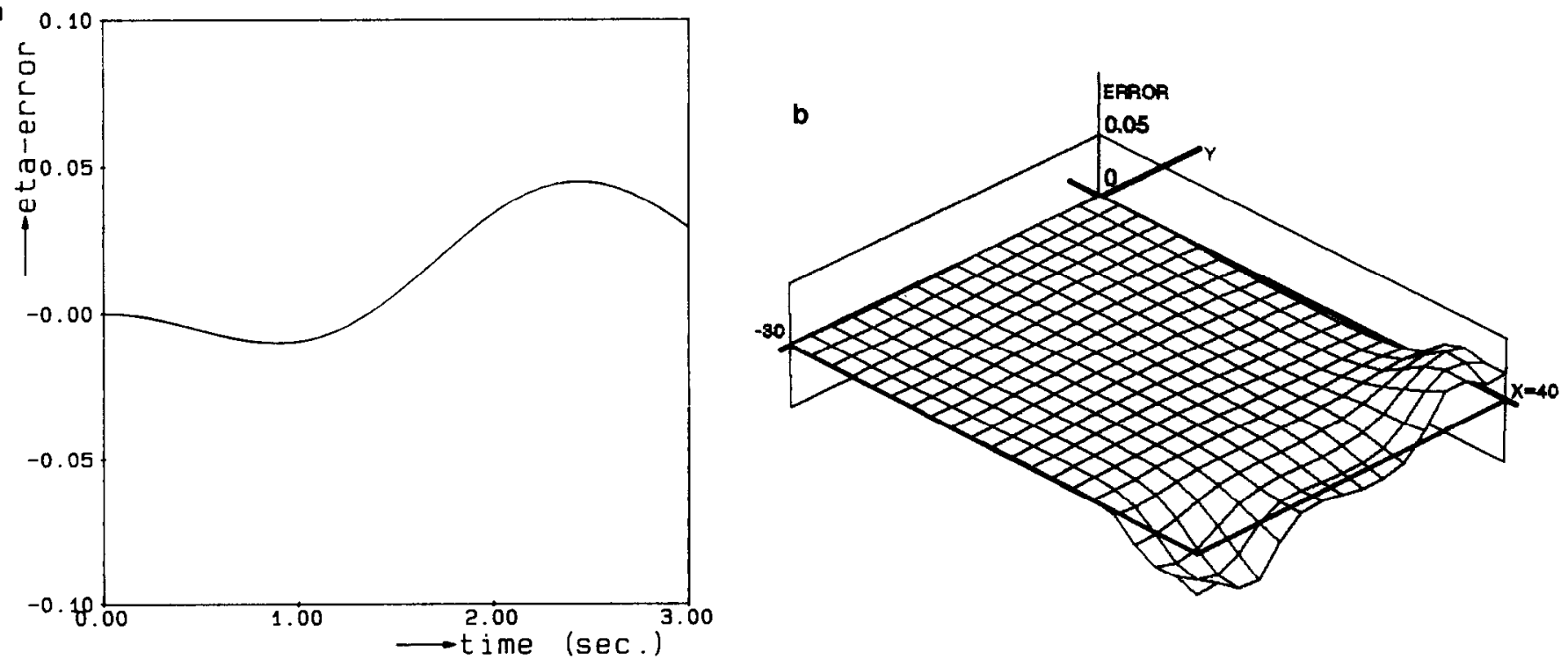

FIG. 9. (a) Eta error in time. Time development of the error in test $6,(x, y, z)=(39,-3,0)$. (b) Errors on the free surface in test 6 after $3 \mathrm{~s}$ due to reflections. 
a

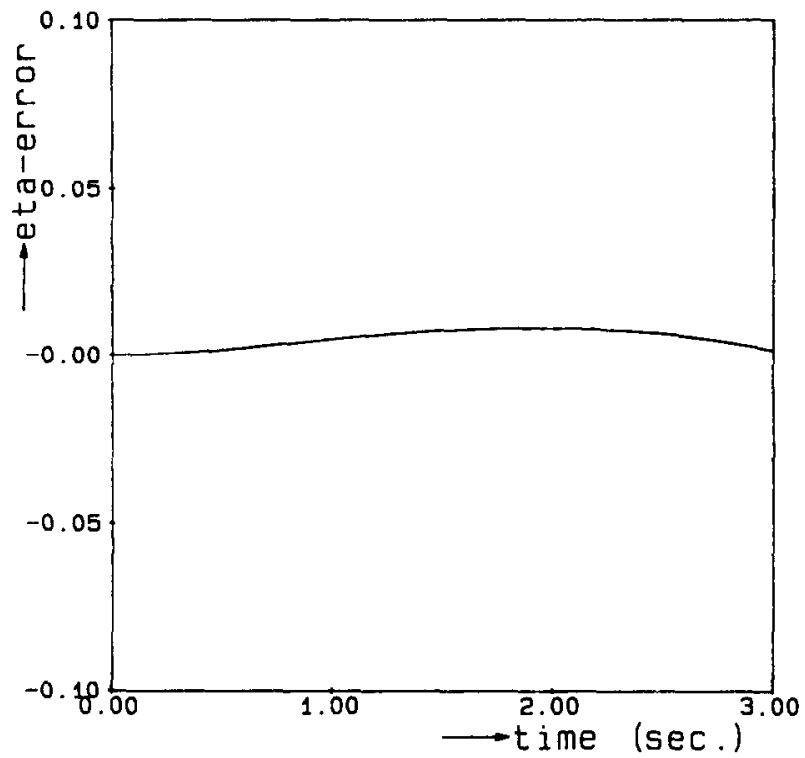

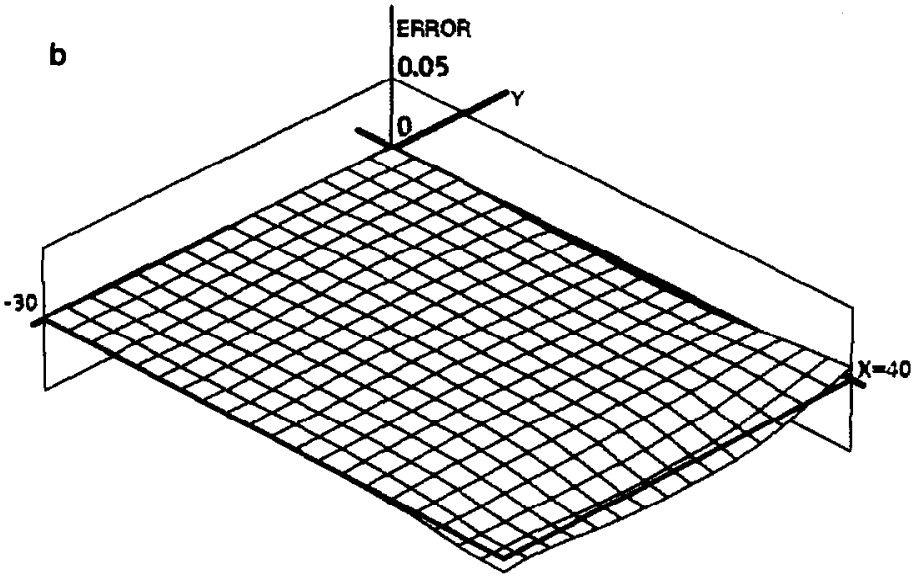

FIG. 10. (a) Eta error in time. Time development of the error in test $7,(x, y, z)=(39,-3,0)$. (b) Errors on the free surface in test 7 after $3 \mathrm{~s}$ due to reduced reflections.

section, with $\theta=0$. These waves can be generated by using Eq. (4.1) on the boundary at $x=0$, with $\alpha$ in every point such that reflected waves from the pile are absorbed (direction of best absorption directed towards $(x, y)=(6,-3))$.

In the first test, first-order absorbing boundary condition are imposed on the boundaries where the waves must leave the domain. The angle of best absorption is directed towards the incoming wave. In the second test, secondorder absorbing boundary conditions are imposed on those boundaries (Eqs. (3.33) and (3.41)). The angles of best absorption $\alpha_{1}$ and $\alpha_{2}$ are chosen to absorb the incoming wave and the reflected wave: $\alpha_{1}$ is chosen to be exact for plane waves from $(x, y)=(8,-4)$ (on the pile), and $\alpha_{2}$ is directed towards the incoming wave. On the pile, the wall condition $(\partial \phi / \partial n=0)$ is imposed.

The analytical solution, used for obtaining the initial values and to check the numerical results, can be found in, for instance, Sarpkaya and Isaacson [11]. The errors in the wave elevation after $2.5 \mathrm{~s}$ are given in Fig. 11.

In Fig. 11a it can be seen that the calculations with a firstorder absorbing boundary condition provide large errors, especially behind the pile. The second-order boundary conditions provide smaller reflections, but still of the same order, although the boundary conditions are such that they should absorb the incoming waves and the diffracted waves well. The small reduction of reflections by using higher
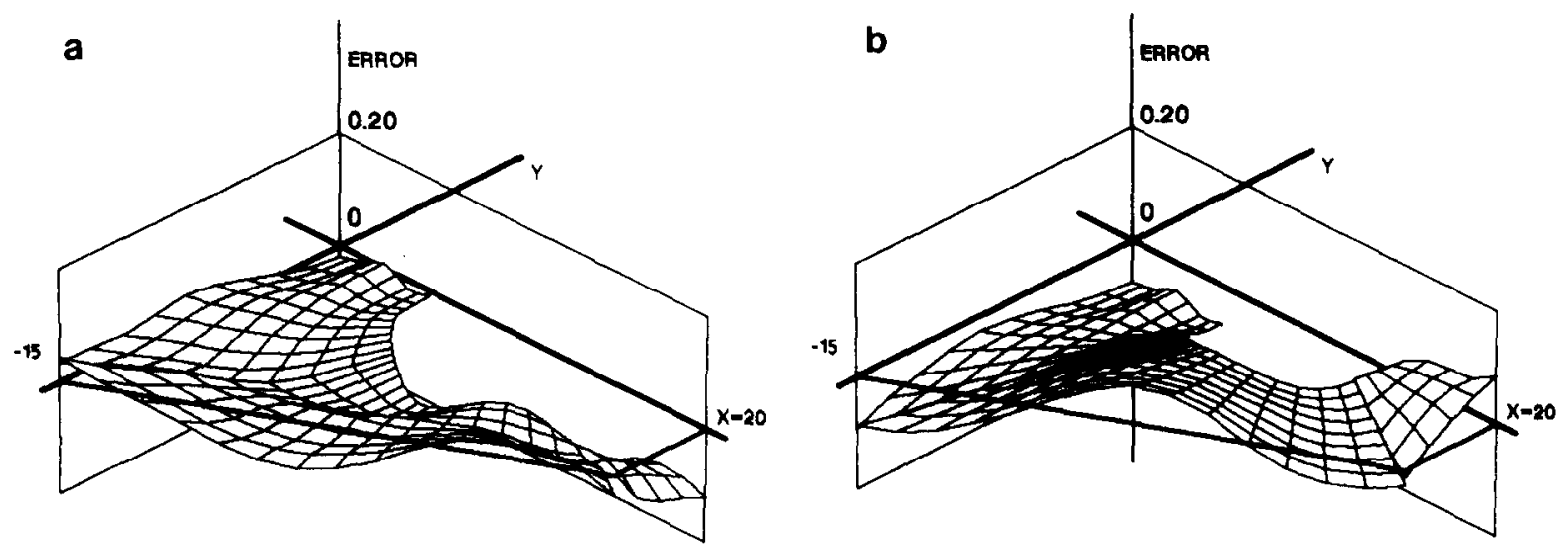

FIG. 11. (a) Errors on the free surface with first-order absorbing boundary conditions. (b) Errors on the free surface with second-order absorbing boundary conditions. 
order absorbing boundary conditions is due to the fact that the diffracted waves are not plane waves. Modifications of the boundary conditions to reduce these errors have not been implemented.

\section{CONCLUSIONS}

We found that the first- and second-order absorbing boundary conditions, imposed on the vertical boundaries of the models yield a stable system. Special (first- or secondorder) conditions must be used in the outmost collocation points for the second-order absorbing boundary condition. The stability was proved with the help of two propositions. Although arguments have been given that support these propositions, they still remain to be proved.

The theoretical stability results are confirmed by numerical experiments.

The reflections that the boundary conditions provide for plane waves are not larger than theoretically expected. The first-order conditions can be used very well when waves from one particular direction have to be absorbed. The second-order condition provides low reflections for waves from different directions.

The proposed absorbing boundary conditions are less well-suited for circular radiating waves. Modifications are needed to improve the absorbing properties of the boundary conditions for such waves.

\section{ACKNOWLEDGMENTS}

The investigations were supported by the Netherlands Technology Foundation (STW), by the Dutch Working-Group on Supercomputer Usage, by Delft Hydraulics, and by the University of Twente.

\section{REFERENCES}

1. W. F. Ames, Numerical Methods for Partial Differential Equations, second edition (Academic Press, Orlando/New York, 1977).

2. J. Broeze, Delft Hydraulics Report No. H475, Marknesse, 1988 (unpublished).

3. R. Courant and D. Hilbert, Methods of Mathematical Physics, II, (Interscience, New York, 1962).

4. B. Engquist and A. Majda, Math. Comput. 31, 629 (1977).

5. B. Engquist and A. Majda, Commun. Pure Appl. Math. 32, 313 (1979).

6. B. Gustafsson, H.-O. Kreiss, and A. Sundstrom, Math. Comput. 26, 649 (1977).

7. R. L. Higdon, SIAM Rev. 28, 177 (1986).

8. J. E. Romate, Ph.D. thesis, University of Twente, Enschede, 1989 (unpublished).

9. J. E. Romate, J. Comput. Phys. 99, 135 (1992).

10. J. E. Romate, J. Eng. Math. 22, 123 (1988).

11. T. Sarpkaya and M. Isaacson, Mechanics of Wave Forces on Offshore Structures (Van Nostrand Reinhold, New York, 1981). 